\title{
Performance of a diagnostic algorithm for fibrotic hypersensitivity pneumonitis. A case-control study
}

Sabina A. Guler ${ }^{1 *} \mathbb{D}$, Eva Wohlfarth ${ }^{1,2}$, Sabina Berezowska3,4, Thomas K. Geiser ${ }^{1}$, Lukas Ebner ${ }^{5}$ and Manuela Funke-Chambour ${ }^{1}$

\begin{abstract}
Background: The differential diagnosis fibrotic hypersensitivity pneumonitis (HP) versus idiopathic pulmonary fibrosis (IPF) is important but challenging. Recent diagnostic guidelines for HP emphasize including multidisciplinary discussion (MDD) in the diagnostic process, however MDD is not comprehensively available.

We aimed to establish the diagnostic accuracy and prognostic validity of a previously proposed HP diagnostic algorithm that foregoes MDD.

Methods: We tested the algorithm in patients with an MDD diagnosis of fibrotic HP or IPF (case control study) and determined diagnostic test performances for diagnostic confidences of $\geq 90 \%$ and $\geq 70 \%$. Prognostic validity was established using Cox proportional hazards models.
\end{abstract}

Results: Thirty-one patients with fibrotic HP and 50 IPF patients were included. The algorithm-derived $\geq 90 \%$ confidence level for HP had high specificity $(0.94,95 \%$ confidence interval [Cl] $0.83-0.99)$, but low sensitivity $(0.35$ [95\% Cl $0.19-0.55]$, J-index 0.29). Test performance was improved for the $\geq 70 \%$ confidence level (J-index 0.64) with a specificity of 0.90 ( $95 \% \mathrm{Cl} 0.78-0.97$ ), and a sensitivity of 0.74 (95\%Cl 0.55-0.88). MDD fibrotic HP diagnosis was strongly associated with lower risk of death (adjusted hazard ratio [HR] 0.10 [0.01-0.92], $p=0.04$ ), whereas the algorithmderived $\geq 70 \%$ and $\geq 90 \%$ confidence diagnoses were not significantly associated with survival (adjusted HR 0.37 $[0.07-1.80], p=0.22$, and adjusted HR $0.41[0.05-3.25], p=0.39$, respectively).

Conclusion: The algorithm-derived $\geq 70 \%$ diagnostic confidence had satisfactory test performance for MDD-HP diagnosis, with insufficient sensitivity for $\geq 90 \%$ confidence. The lowest risk of death in the MDD-derived HP diagnosis validates the reference standard and suggests that a diagnostic algorithm not including MDD, might not replace the latter.

Keywords: Pulmonary Fibrosis, Environmental Exposure, Algorithms, Diagnosis, Alveolitis, Extrinsic Allergic

*Correspondence: sabina.guler@insel.ch

1 Department of Pulmonary Medicine, Inselspital, Bern University Hospital, University of Bern, 3010 Bern, Switzerland

Full list of author information is available at the end of the article

\begin{abstract}
Background
Interstitial lung diseases (ILD) lead to significant symptoms, physical impairment, and early mortality $[1,2]$. Fibrotic hypersensitivity pneumonitis (HP) is an ILD subtype caused by an ongoing or repetitive exposure to an inhaled antigen in sensitised and susceptible individuals [1]. The identification of patients with HP is crucial, particularly to prevent ongoing exposure to the causative
\end{abstract} original author(s) and the source, provide a link to the Creative Commons licence, and indicate if changes were made. The images or other third party material in this article are included in the article's Creative Commons licence, unless indicated otherwise in a credit line to the material. If material is not included in the article's Creative Commons licence and your intended use is not permitted by statutory regulation or exceeds the permitted use, you will need to obtain permission directly from the copyright holder. To view a copy of this licence, visit http://creativecommons.org/licenses/by/4.0/. The Creative Commons Public Domain Dedication waiver (http://creativeco mmons.org/publicdomain/zero/1.0/) applies to the data made available in this article, unless otherwise stated in a credit line to the data. 
antigen. However, in chronic cases where an inciting antigen cannot be identified [3], distinguishing fibrotic HP from idiopathic pulmonary fibrosis (IPF) is challenging with considerable diagnostic overlap between these fibrotic ILD subtypes [4, 5]. Patients with IPF have a worse prognosis than patients with fibrotic HP [6], and the immunosuppressive therapy that is frequently used in HP can be detrimental for IPF patients [7]. This emphasizes the importance of the differential diagnosis fibrotic HP versus IPF.

Due to the heterogeneity in clinical presentation among patients with fibrotic HP and IPF there is no single diagnostic test for accurate discrimination. The recent diagnostic guidelines for HP emphasize the importance of multidisciplinary discussion (MDD), and include MDD in the proposed diagnostic approach [5]. However MDDs are time and resource consuming and not widely available [8], simpler, accurate tools for HP diagnosis are urgently needed. A diagnostic HP algorithm that foregoes MDD has been developed by Morisset and group, with consensus criteria that were identified by a structured Delphi approach [9].

The main objective of this case control study was to validate the algorithm for the differential diagnosis fibrotic HP versus IPF. We aimed to establish the diagnostic accuracy of the algorithm, and to determine the prognostic validity of the algorithm and MDD derived HP diagnoses.

\section{Methods}

\section{Study population, setting, and measurements}

Patients with fibrotic ILD are prospectively recruited within our ongoing cohort study (Swiss Ethics Committee, Bern, KEK 246/15 PB_2016-01524). For this retrospective case control study, we included consecutive patients who were diagnosed with either fibrotic HP (cases) or IPF (controls) in our MDD between 03/2013 and $11 / 2018$. Patients were included if the necessary data for a confident MDD diagnosis was available, including exposure history, high resolution computed tomography (HRCT) chest scans, and cytological or histological results if bronchoalveolar lavage (BAL) or lung biopsy were performed. All patients were questioned regarding exposure to a potentially inciting antigen using a questionnaire customized to our local practice. Antigen exposures that were considered relevant for HP included mould, hay, agricultural dusts, bird feathers and droppings, and the use of a hot tub, swimming pool, or air humidifier.

Aiming to evaluate the diagnostic accuracy and prognostic validity of a fibrotic HP diagnostic algorithm [9], we included patients with IPF as a control group, since IPF is the most challenging differential diagnosis. We defined MDD diagnosis as our reference standard [1, 2, 5, 8]. As previously described, our MDD meetings include experienced chest radiologists, pathologists, and specialized ILD physicians $[8,10]$. For the purpose of this study, a chest radiologist who was blinded for the diagnosis (LE) reanalysed the chest CT scans of all IPF and fibrotic HP cases.

Patient demographics and baseline characteristics were collected from the registry. All measurements were accomplished within 3 months of MDD diagnosis. Pulmonary function tests were conducted according to established protocols $[11,12]$. We calculated the Composite Physiologic Index (CPI), which was developed to predict radiological extent of fibrosis by aggregating forced vital capacity (FVC), forced expiratory volume in $1 \mathrm{~s}$ (FEV1), and diffusing capacity of the lung for carbon monoxide (DLCO).[13] Serum IgG against specific antigens were measured in some patients, however results were not included in the proposed algorihm [9], and not considered for this study.

\section{Algorithm for the diagnosis of fibrotic HP}

We applied a slightly adapted version of the HP diagnostic algorithm proposed by Morisset and group [9]. Every case was approached in a stepwise manner as outlined in the algorithm, starting with stratification by exposure to an inciting antigen (Fig. 1). As proposed, a HRCT pattern was considered typical for HP if either a combination of mosaic attenuation, ground-glass and normal lung, or a combination of mosaic attenuation and signs of fibrosis were present [9]. Usual interstitial pneumonia (UIP) and probable UIP were classified based on the most recent diagnostic criteria for IPF [2]. This in slight contrast to the Morisset algorithm, which included possible instead of probable UIP [14]. We also included other HRCT patterns as a third category, since in our cohort some HRCT pattern were neither classified as (probable) UIP nor as typical for HP. The threshold for lymphocytosis in BAL was set at $40 \%$ as suggested [9]. The histopathological features for HP included chronic bronchiolocentric inflammation, poorly formed non-necrotizing granulomas, giant cells, airway-centred interstitial fibrosis, and the absence of an alternative diagnosis [15]. We considered provisional diagnosis with high confidence (diagnostic confidence 70-89\%) and a confident diagnosis (diagnostic confidence $\geq 90 \%$ ) as diagnostically meaningful for the differential diagnosis fibrotic HP versus IPF. Low confidence diagnosis (diagnostic confidence 50-69\%) was not evaluated for diagnostic test performance $[9,16]$.

\section{Statistical analysis}

Baseline patient data are reported as mean (standard deviation) or median (interquartile range). 


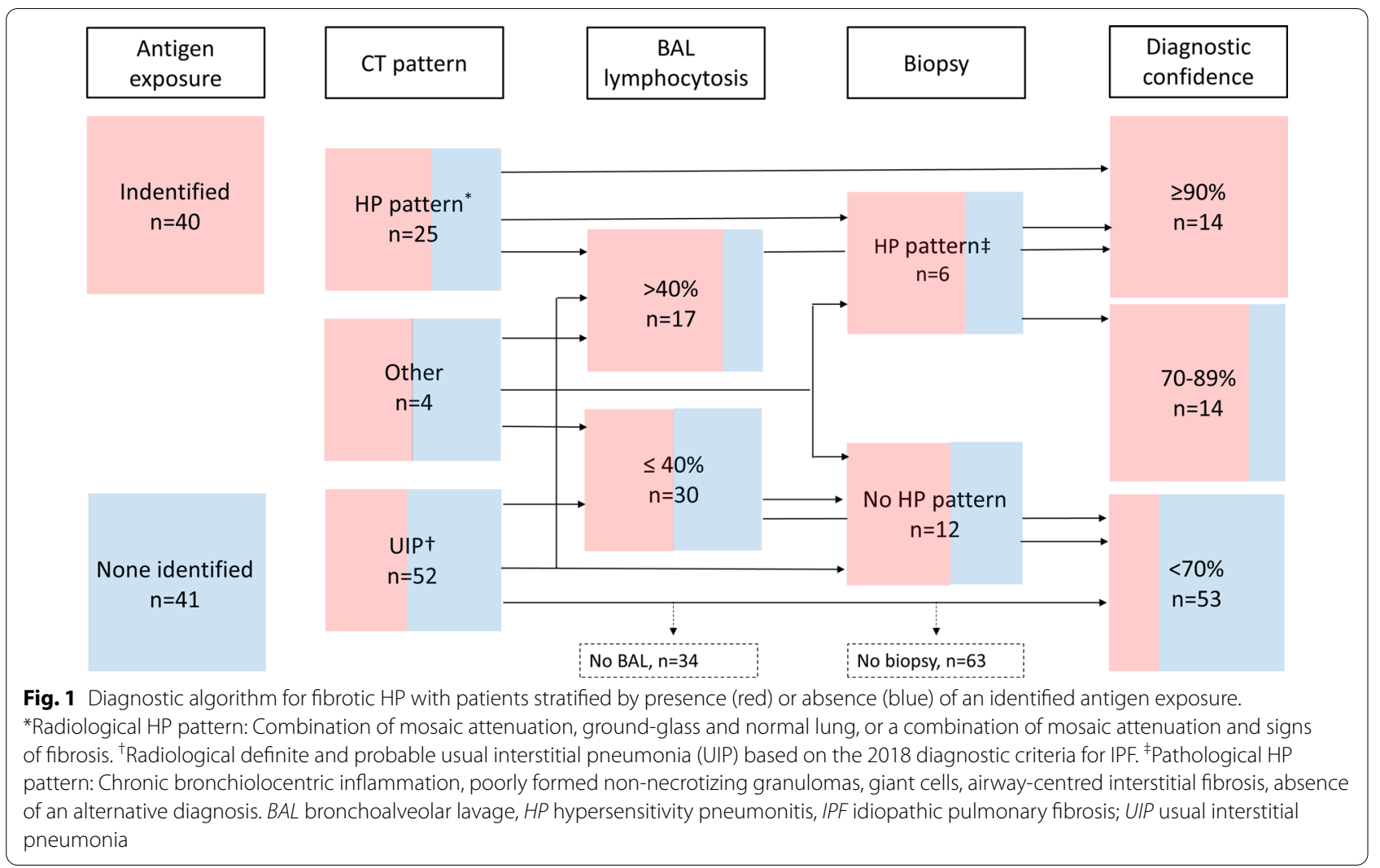

Diagnostic test characteristics with $95 \%$ confidence intervals $(95 \% \mathrm{CI})$ were calculated for an algorithm derived diagnostic confidence $\geq 90 \%$ and $\geq 70 \%$ with an MDD diagnosis of fibrotic HP as reference standard. Sensitivity, specificity, positive and negative predictive values (PPV, NPV) were derived from two-by-two tables. Youden's $(\mathrm{J})$ index summarizes sensitivity and specificity, and is thus affected by disease prevalence, whereas diagnostic accuracy is the proportion of correctly classified cases among all cases, and not affected by disease prevalence. Based on the assumption that patients with a "true" diagnosis of fibrotic HP have a more favorable prognosis than patients with IPF $[6$, 17], we determined the prognostic validity of the MDD diagnosis and the algorithm derived diagnosis. Cox proportional hazards models were used to determine risk of mortality associated with baseline variables and fibrotic HP diagnoses (MDD versus algorithm derived). Adjusted models included radiological definite or probable UIP pattern, and CPI to account for potential confounding by ILD severity. Harrell's C-statistic was used to indicate discrimination of the models. The proportional hazards assumption for all Cox regression models was tested based on weighted residuals. A two-sided $\mathrm{p}<0.05$ was used to indicate statistical significance.
Data were analysed using $R$ version 3.6.0 (R Foundation for Statistical Computing, Vienna, Austria) [18].

\section{Results}

Thirty-one and 50 patients with an MDD diagnosis of fibrotic HP or IPF respectively were included in this study (Table 1). $78 \%$ of the cohort were men with a mean

Table 1 Baseline characteristics by MDD diagnoses

\begin{tabular}{lcc}
\hline & $\begin{array}{l}\text { Hypersensitivity } \\
\text { pneumonitis } \\
\mathbf{n = 3 1}\end{array}$ & $\begin{array}{l}\text { Idiopathic } \\
\text { pulmonary fibrosis } \\
\mathbf{n}=\mathbf{5 0}\end{array}$ \\
\hline Age, years & $64.0(10.7)$ & $68.4(9.5)$ \\
Sex, men & $15(48 \%)$ & $48(96 \%)$ \\
Ever smoker & $12(39 \%)$ & $38(76 \%)$ \\
Smoked pack years & $20(12.5-50)$ & $30(20-40)$ \\
Body mass index, kg/m² & $27.9(5.4)$ & $27.2(4.5)$ \\
FVC, \%-predicted & $73(23)$ & $65(17)$ \\
FEV1, \%-predicted & $74(18)$ & $70(17)$ \\
FEV1/FVC, \% & $103(9)$ & $108(7)$ \\
DLCO, \%-predicted & $55(20)$ & $48.5(13.8)$ \\
Composite Physiologic & $42.0(15.5)$ & \\
Index & & \\
\hline
\end{tabular}

DLCO diffusing capacity of the lung for carbon monoxide, FEV1 forced vital capacity in $1 \mathrm{~s}, F V C$ forced vital capacity, MDD multidisciplinary discussion 
(standard deviation) age of 67 (10) years, and a moderately reduced FVC of 68 (19) \%-predicted, and a DLCO of 51 (20) \%-predicted. 40 patients (49\%) reported exposures to potentially causative antigens $(74 \%$ and $34 \%$ of MDD HP and IPF patients, respectively). A radiological probable or definite UIP pattern was present in $29 \%$ and $86 \%$ of MDD HP and IPF patients, respectively, with $68 \%$ of the HP and $8 \%$ of the IPF patients showing a radiological pattern compatible with HP. Of the patients with MDD diagnosed HP 26 (84\%) had BAL available with BAL lymphocytosis present in 14 of these cases. BAL was available in 21 (42\%) of MDD diagnosed IPF cases, with lymphocytosis in 3 patients. Surgical lung biopsy was available in $22 \%$ of IPF and $23 \%$ of HP patients.

\section{Diagnostic accuracy}

A diagnostic confidence for fibrotic HP of $\geq 90 \%$ and a diagnostic confidence $70-89 \%$ were reached in 14 cases each (Fig. 1). The algorithm-derived $\geq 90 \%$ confidence level had a high specificity (0.94 [95\% CI 0.83-0.99]) but low sensitivity ( 0.35 [95\% CI $0.19-0.55]$ ) for MDD diagnosis of fibrotic HP, with an overall insufficient test performance (J-index 0.29 [95\% CI $0.03-0.53]$ ). The algorithm-derived $\geq 70 \%$ confidence level showed higher overall test performance (J-index 0.64 [95\% CI

Table 2 Diagnostic test characteristics for algorithm derived HP diagnoses

\begin{tabular}{lll}
\hline & \multicolumn{2}{l}{$\begin{array}{l}\text { Algorithm derived diagnostic } \\
\text { confidence }\end{array}$} \\
\cline { 2 - 3 } & $\geq \mathbf{9 0 \%}$ & $\geq \mathbf{7 0 \%}$ \\
\hline Sensitivity $(95 \% \mathrm{Cl})$ & $0.35(0.19-0.55)$ & $0.74(0.55-0.88)$ \\
Specificity $(95 \% \mathrm{Cl})$ & $0.94(0.83-0.99)$ & $0.90(0.78-0.97)$ \\
J-index $(95 \% \mathrm{Cl})$ & $0.29(0.03-0.53)$ & $0.64(0.34-0.85)$ \\
PPV $(95 \% \mathrm{Cl})$ & $0.79(0.49-0.95)$ & $0.82(0.63-0.94)$ \\
NPV (95\% Cl) & $0.70(0.58-0.81)$ & $0.85(0.72-0.93)$ \\
Diagnostic accuracy & $0.71(0.60-0.81)$ & $0.84(0.74-0.91)$ \\
\hline
\end{tabular}

Cl confidence interval, HP hypersensitivity diagnosis, J-index Youden's index, NPV negative predictive value, $P P V$ positive predictive value
0.34-0.85]) with improved sensitivity (0.74 [95\% CI 0.55$0.88]$ ), positive $(0.82$ [95\% CI $0.63-0.94])$, and negative predictive values $(0.85$ [95\% CI $0.72-0.93])$, with marginally lower specificity (0.90 [95\% CI 0.78-0.97]), (Table 2).

\section{Prognostic validity}

Over a median time of 16 months (interquartile range 6-30), 28 patients deceased.

Per one \% increase in DLCO patients had a $5 \%$ decrease in risk of death (hazard ratio [HR] 0.95, 95\% CI 0.92-0.98, $p=0.007)$. FVC \%-predicted was borderline associated with mortality (HR $0.98,95 \%$ CI $0.96-1.00, p=0.07$ ), and CPI was significantly associated with mortality (HR $1.06,95 \%$ CI $1.01-1.10, p=0.01)$. Patients with a definite/ probable radiological UIP pattern had a 5.4 higher risk of death (95\% CI 1.62-17.8, $p=0.006)$. Age, sex, smoking status, body mass index, identification of the inciting antigen, and availability of surgical lung biopsy were not associated with survival.

MDD diagnosis of fibrotic HP was strongly associated with a lower risk of death on unadjusted analysis and with adjustment for potential confounding by disease severity (CPI) and a radiological UIP pattern (HR 0.10 [0.01-0.92], $p=0.04$ ). The algorithm derived $\geq 70 \%$ confidence diagnosis showed a significant association with lower risk of death on unadjusted analysis, which lost statistical significance on adjusted analysis (HR 0.37 $[0.07-1.80], p=0.22)$. The algorithm derived $\geq 90 \%$ confidence diagnosis was not significantly associated with survival on unadjusted and adjusted analysis (HR 0.41 [0.05-3.25], $p=0.39$ ), (Table 3, Fig. 2).

\section{Discussion}

With this case control study, we validate a previously developed diagnostic algorithm for fibrotic HP [9], by assessing its diagnostic test performance in a cohort of patients with fibrotic HP and a control cohort of patients with IPF, using MDD as the reference standard. The algorithm derived diagnostic confidence $\geq 70 \%$ demonstrated a high specificity, acceptable sensitivity, and a diagnostic

Table 3 Prognostic validity of MDD and algorithm derived HP diagnoses

\begin{tabular}{|c|c|c|c|c|c|c|}
\hline & \multicolumn{3}{|c|}{ Unadjusted analyses } & \multicolumn{3}{|c|}{ Adjusted for CPI and UIP } \\
\hline & HR $(95 \% \mathrm{Cl})$ & $p$-value & C-index & $\mathrm{HR}(95 \% \mathrm{Cl})$ & $p$-value & C-index \\
\hline \multicolumn{7}{|c|}{ Multidisciplinary discussion diagnosis for fibrotic HP } \\
\hline HP & $0.06(0.01-0.45)$ & 0.006 & 0.68 & $0.10(0.01-0.92)$ & 0.04 & 0.76 \\
\hline \multicolumn{7}{|c|}{ Algorithm derived diagnosis for fibrotic HP } \\
\hline$\geq 90 \%$ confidence & $0.16(0.02-1.20)$ & 0.07 & 0.58 & $0.41(0.05-3.25)$ & 0.39 & 0.72 \\
\hline$\geq 70 \%$ confidence & $0.14(0.03-0.59)$ & 0.007 & 0.65 & $0.37(0.07-1.80)$ & 0.22 & 0.72 \\
\hline
\end{tabular}

C-index Harrell's C-statistic, HP hypersensitivity pneumonitis, HR hazard ratio, UIP radiological usual interstitial pneumonia pattern 


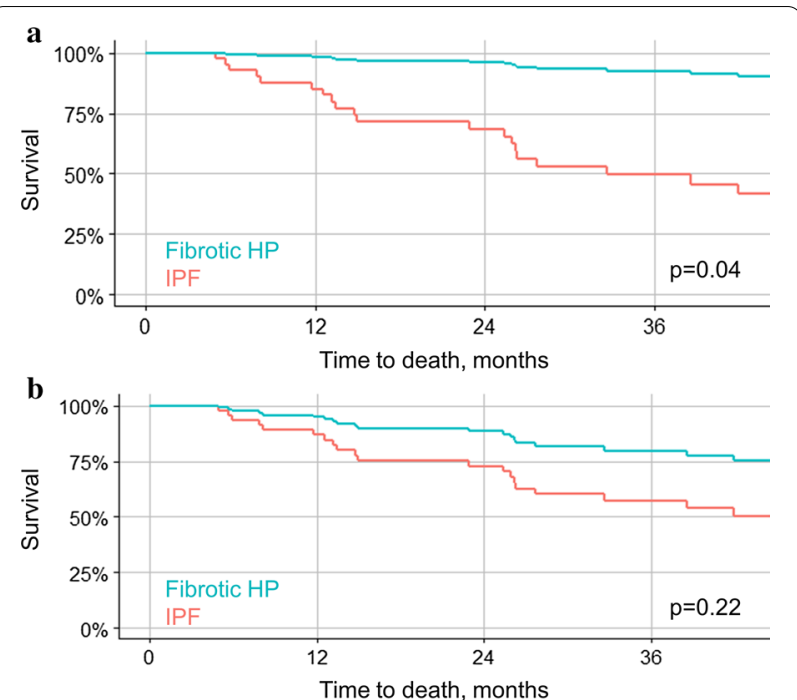

Fig. 2 Survival by multidisciplinary discussion a and algorithm derived $\geq 70 \%$ diagnostic confidence $\mathbf{b}$ diagnoses for fibrotic HP versus IPF. Survival curves from Cox Proportional Hazard models adjusted for Composite Physiological Index and radiological pattern of definite or probable UIP pattern. HP hypersensitivity pneumonitis, IPF idiopathic pulmonary fibrosis, UIP usual interstitial pneumonia

accuracy of $84 \%$. An algorithm derived diagnostic confidence level $\geq 90 \%$ showed poor sensitivity and lower diagnostic accuracy (71\%) with an expectedly higher specificity than the $\geq 70 \%$ confidence level. Overall, if a confidence level $\geq 70 \%$ was considered diagnostic for fibrotic HP, the algorithm performed well in our cohort, whereas diagnostic performance for the $\geq 90 \%$ threshold was insufficient.

MDD diagnosis of fibrotic HP showed a stronger association with survival than the algorithm derived diagnoses on unadjusted analysis, and with adjustment for disease severity and UIP pattern, which have been shown to impact survival in IPF and fibrotic HP beyond diagnosis $[6,17]$. This observation strengthens the validity of MDD diagnosis as the reference standard in our cohort.

Identification of the inciting antigen is crucial for HP diagnosis and management. In patients with an identified antigen diagnostic confidence for HP is higher [9], the danger of IPF misclassification is lower [4], and prognosis is more favorable [3]. However, in less than $50 \%$ of patients an antigen can be identified [3]. We confirm that a systematic exposure assessment is helpful for antigen identification. With a questionnaire tailored to local practice we identified an antigen exposure in $74 \%$ of $\mathrm{HP}$ cases, but also in $34 \%$ of IPF cases, which underlines the low specificity of patient-reported antigen exposure, and the need for estimation of potential causality by the clinician [19]. Testing of specific serum IgG levels can confirm exposure to the antigen at some point in the patient's life, without prove of causality to the ILD [5].

BAL lymphocytosis is important for HP diagnosis, either as a piece of information to the MDD or as a formal diagnostic criterion or integrated in an algorithm. However, the optimal BAL lymphocytosis cut-off still needs to be determined: A recent meta-analysis demonstrated an optimized sensitivity (71\%) and specificity (68\%) for a $21 \%$ lymphocytosis threshold [20], and a threshold of $20 \%$ has been proposed to decide if a surgical lung biopsy for suspected HP is needed [21]. Conversely, most ILD experts indicated that they find a threshold of $40 \%$ to be useful for the diagnosis of chronic HP [9], and experts contributing to the new diagnostic guidelines indicated that they find a threshold of $30 \%$ reasonable [5]. Likely different thresholds are valid for different positions of BAL in a diagnostic algorithm.

Recent guidelines suggested BAL, transbronchial cryobiopsy, and surgical lung biopsy for the diagnosis of fibrotic HP with very low confidence [5]. In 2 (6\%) of our patients with an MDD diagnosis of HP and in 19 (35\%) of the MDD diagnosed IPF controls, no surgical lung biopsy or BAL was performed. In the individual patient, the risk-benefit ratio of these invasive procedures is challenging to estimate. Aside from the diagnostic confidence before biopsy (which can be estimated with a diagnostic algorithm), the individual procedural risk likely determines the role of biopsy in the diagnostic algorithm. Consequently, a diagnostic HP algorithm might need tailoring for patients with advanced disease, important comorbidities, and frailty.

The multi-component diagnostic process emphasizes the importance of MDDs for diagnostic decision making in complex cases. The overlap between fibrotic ILDs and the heterogeneity within fibrotic HP and IPF might not be sufficiently accounted for by an algorithm in every case, and MDD discussion likely provides additional granularity for the assessment of complex cases. The prognostic validity of chronic HP MDD diagnosis in this study further supports this approach. In line, the recent diagnostic guidelines for HP confirm the central role of MDD in the diagnostic evaluation of patients with possible HP [5].

Acknowledging the limited availability of resources for MDD, a diagnostic algorithm might be valuable to preselect typical cases where MDD discussion is less important. Future studies might investigate the implementation of diagnostic decision trees including the option of MDD discussion. Our findings suggest that an algorithm might be a valid tool for fibrotic HP diagnosis. The applicability of the algorithm might further be improved with adaptations according to local practice. Furthermore, future integration new diagnostic tools, e.g. blood-based 
biomarkers and genomic classifiers might improve the discriminative ability of diagnostic algorithms [22, 23].

Our local single centre study limits generalizability of the findings to populations with similar demographics and antigen exposure patterns. In our experience IPF is the most challenging and impactful differential diagnosis of fibrotic HP. However, our results do not apply for other differential diagnoses. A recent Japanese study reported the chronic HP algorithm to be useful in a heterogeneous ILD cohort, with potential avoidance of surgical lung biopsy in some of their ILD patients. However, 59\% of cases were excluded from the algorithm, mainly because they did not have radiological features consistent with UIP or with HP [24]. By adding the "other radiological pattern" category to the algorithm, we ensured that every case was captured by the algorithm. Furthermore, prevalence of fibrotic ILD subtypes varies between cohorts, which can impact on PPV, NPV, and diagnostic accuracy. Sensitivity, specificity, and Youden's index are not influenced by disease prevalence. Our adaptations to the proposed algorithm were minor, but necessary to include all cases and controls in the algorithm. With the application of a customized antigen exposure questionnaire and detailed interviews, we tried to capture all relevant exposures, however missing exposures are still possible. Incorporation bias is an inherent problem to many diagnostic test studies in ILD. The diagnostic pieces of the algorithm are also included in MDD discussions, which likely inflates the agreement between the two assessments. Furthermore, there is no "gold standard" for fibrotic HP diagnosis. We tried to account for this by demonstrating the prognostic validity of our reference standard.

\section{Conclusion}

Current diagnostic guidelines for HP recommend a diagnostic approach that includes MDD [5]. In this study, we validate a proposed diagnostic algorithm for fibrotic HP that foregoes MDD. We demonstrate acceptable test performance of the $\geq 70 \%$ diagnostic confidence level compared to the reference standard MDD, and consequently endorse the incorporation of algorithms to fibrotic HP diagnosis. Due to the low sensitivity and potential danger of missing HP cases, we suggest not relying on the current algorithm's $\geq 90 \%$ diagnostic confidence level for the differential diagnosis HP versus IPF. Algorithms likely need to be adapted to regionally distinct exposures, local practice, and availability of diagnostic tests, with prospective validation in corresponding cohorts. Furthermore, algorithms might not be sufficiently accurate for complex cases and are unlikely to replace MDDs entirely.

\section{Abbreviations}

BAL: Bronchoalveolar lavage; Cl: Confidence interval; C-index: Harrell's C-statistic; CPI: Composite Physiologic Index; DLCO: Diffusing capacity of the lung for carbon monoxide; FEV1: Forced expiratory volume in $1 \mathrm{~s}$; FVC: Forced vital capacity; HP: Hypersensitivity pneumonitis; HR: Hazard ratio; HRCT: High resolution computed tomography; ILD: Interstitial lung disease; IPF: Idiopathic pulmonary fibrosis; J-index: Youden's index; MDD: Multidisciplinary discussion; NPV: Negative predictive value; NSIP: Nonspecific interstitial pneumonia; PPV: Positive predictive value; UIP: Usual interstitial pneumonia.

\section{Acknowledgements \\ We thank the patients of our ILD clinic who contribute to the Swiss IIP registry. We acknowledge Michèle Tschiemer for her work and support as ILD nurse.}

\section{Authors' contributions}

SAG and MF designed the study. SAG, EW, SB, TKG, LE, and MF contributed to data acquisition, analysis, and interpretation of the data. SAG, EW, and MF drafted the manuscript. All authors revised the manuscript for important intellectual content and approved the final version. All authors read and approved the final manuscript.

\section{Funding}

The Swiss IIP registry is funded by unrestricted grants from Boehringer Ingelheim and Roche.

\section{Availability of data and materials}

The datasets used and analysed during the current study are available from the corresponding author on reasonable request.

\section{Declarations}

Ethics approval and consent to participate

All participants provided written informed consent for participation in the current study (Swiss Ethics Committee, Bern, KEK 246/15 PB_2016-01524).

\section{Competing interests}

The authors report no relevant conflicts of interest related to this work.

\section{Author details}

'Department of Pulmonary Medicine, Inselspital, Bern University Hospital, University of Bern, 3010 Bern, Switzerland. ${ }^{2}$ Schlosspraxis Schwarzenburg, Schwarzenburg, Switzerland. ${ }^{3}$ Institute of Pathology, University of Bern, Bern, Switzerland. ${ }^{4}$ Department of Laboratory Medicine and Pathology, Institute of Pathology, Lausanne University Hospital and Lausanne University, Lausanne, Switzerland. ${ }^{5}$ Department of Diagnostic, Interventional and Pediatric Radiology, Inselspital, Bern University Hospital, University of Bern, Bern, Switzerland.

Received: 8 November 2020 Accepted: 18 April 2021

Published online: 23 April 2021

\section{References}

1. Travis WD, Costabel U, Hansell DM, King TE Jr, Lynch DA, Nicholson AG, Ryerson CJ, Ryu JH, Selman M, Wells AU, et al. An official American Thoracic Society/European Respiratory Society statement: update of the international multidisciplinary classification of the idiopathic interstitial pneumonias. Am J Respir Crit Care Med. 2013;188:733-48.

2. Raghu G, Remy-Jardin M, Myers JL, Richeldi L, Ryerson CJ, Lederer DJ, Behr J, Cottin V, Danoff SK, Morell F, et al. Diagnosis of idiopathic pulmonary fibrosis. An official ATS/ERS/JRS/ALAT clinical practice guideline. Am J Respir Crit Care Med. 2018;198:e44-68.

3. Fernandez Perez ER, Swigris JJ, Forssen AV, Tourin O, Solomon JJ, Huie TJ, Olson AL, Brown KK. Identifying an inciting antigen is associated with improved survival in patients with chronic hypersensitivity pneumonitis. Chest. 2013;144:1644-51.

4. Morell F, Villar A, Montero MA, Munoz X, Colby TV, Pipvath S, Cruz MJ, Raghu G. Chronic hypersensitivity pneumonitis in patients diagnosed with idiopathic pulmonary fibrosis: a prospective case-cohort study. Lancet Respir Med. 2013;1:685-94. 
5. Raghu G, Document S, Remy-Jardin M, Ryerson CJ, Myers JL, Kreuter M, Vasakova M, Bargagli E, Chung JH, Collins BF, et al. Diagnosis of hypersensitivity pneumonitis in adults: an official ATS/JRS/ALAT clinical practice guideline. Am J Respir Crit Care Med. 2020. https://doi.org/10.1164/rccm. 202005-2032ST.

6. Ryerson CJ, Vittinghoff E, Ley B, Lee JS, Mooney JJ, Jones KD, Elicker BM, Wolters PJ, Koth LL, King TE Jr, Collard HR. Predicting survival across chronic interstitial lung disease: the ILD-GAP model. Chest. 2014:145:723-8.

7. Idiopathic Pulmonary Fibrosis Clinical Research N, Raghu G, Anstrom KJ, King TE, Lasky JA, Martinez FJ. Prednisone, azathioprine, and N-acetylcysteine for pulmonary fibrosis. N Engl J Med. 2012;366:1968-77.

8. Walsh SL, Wells AU, Desai SR, Poletti V, Piciucchi S, Dubini A, Nunes H, Valeyre D, Brillet PY, Kambouchner M, et al. Multicentre evaluation of multidisciplinary team meeting agreement on diagnosis in diffuse parenchymal lung disease: a case-cohort study. Lancet Respir Med. 2016;4:557-65.

9. Morisset J, Johannson KA, Jones KD, Wolters PJ, Collard HR, Walsh SLF, Ley B. Identification of diagnostic criteria for chronic hypersensitivity pneumonitis: an International Modified Delphi Survey. Am J Respir Crit Care Med. 2018:197:1036-44.

10. Guler SA, Berezowska SA, Christe A, Geiser T, Funke-Chambour M. Multidisciplinary discussion for diagnosis of interstitial lung disease in real life. Swiss Med Wkly. 2016;146:W14318.

11. Macintyre N, Crapo RO, Viegi G, Johnson DC, van der Grinten CP, Brusasco V, Burgos F, Casaburi R, Coates A, Enright P, et al. Standardisation of the single-breath determination of carbon monoxide uptake in the lung. Eur Respir J. 2005;26:720-35.

12. Wanger J, Clausen JL, Coates A, Pedersen OF, Brusasco V, Burgos F, Casaburi R, Crapo R, Enright P, van der Grinten CP, et al. Standardisation of the measurement of lung volumes. Eur Respir J. 2005;26:511-22.

13. Wells AU, Desai SR, Rubens MB, Goh NS, Cramer D, Nicholson AG, Colby TV, du Bois RM, Hansell DM. Idiopathic pulmonary fibrosis: a composite physiologic index derived from disease extent observed by computed tomography. Am J Respir Crit Care Med. 2003;167:962-9.

14. Raghu G, Collard HR, Egan JJ, Martinez FJ, Behr J, Brown KK, Colby TV, Cordier JF, Flaherty KR, Lasky JA, et al. An official ATS/ERS/JRS/ALAT statement: idiopathic pulmonary fibrosis: evidence-based guidelines for diagnosis and management. Am J Respir Crit Care Med. 2011;183:788-824.

15. Miller R, Allen TC, Barrios RJ, Beasley MB, Burke L, Cagle PT, Capelozzi VL, Ge Y, Hariri LP, Kerr KM, et al. Hypersensitivity pneumonitis a perspective from members of the pulmonary pathology society. Arch Pathol Lab Med. 2018;142:120-6.
16. Ryerson CJ, Corte TJ, Lee JS, Richeldi L, Walsh SLF, Myers JL, Behr J, Cottin $V$, Danoff SK, Flaherty KR, et al. A standardized diagnostic ontology for fibrotic interstitial lung disease an international working group perspective. Am J Respir Crit Care Med. 2017;196:1249-54.

17. Salisbury ML, Gu T, Murray S, Gross BH, Chughtai A, Sayyouh M, Kazerooni EA, Myers JL, Lagstein A, Konopka KE, et al. Hypersensitivity pneumonitis: radiologic phenotypes are associated with distinct survival time and pulmonary function trajectory. Chest. 2019;155:699-711.

18. R Core Team. R: A language and environment for statistical computing. 2019. https://www.R-project.org/. Accessed 19 Mar 2021.

19. Barnes H, Morisset J, Molyneaux P, Westall G, Glaspole I, Collard HR. A systematically derived exposure assessment instrument for chronic hypersensitivity pneumonitis. Chest. 2020. https://doi.org/10.1016/j.chest. 2019.08.1507.

20. Adderley N, Humphreys CJ, Barnes H, Ley B, Premji ZA, Johannson KA. Bronchoalveolar lavage fluid lymphocytosis in chronic hypersensitivity pneumonitis: a systematic review and meta-analysis. Eur Respir J. 2020. https://doi.org/10.1183/13993003.00206-2020.

21. Salisbury ML, Myers JL, Belloli EA, Kazerooni EA, Martinez FJ, Flaherty KR. Diagnosis and treatment of fibrotic hypersensitivity pneumonia. Where we stand and where we need to go. Am J Respir Crit Care Med. 2016. https://doi.org/10.1164/rccm.201608-1675PP.

22. Richeldi L, Scholand MB, Lynch DA, Colby TV, Myers JL, Groshong SD, Chung JH, Benzaquen S, Nathan SD, Davis JR, et al. Utility of a molecular classifier as a complement to HRCT to identify usual interstitial pneumonia. Am J Respir Crit Care Med. 2020. https://doi.org/10.1164/rccm. 202003-08770C

23. Okamoto T, Fujii M, Furusawa H, Tsuchiya K, Miyazaki Y, Inase N. The usefulness of KL- 6 and SP-D for the diagnosis and management of chronic hypersensitivity pneumonitis. Respir Med. 2015;109:1576-81.

24. Takei R, Yamano Y, Kataoka K, Yokoyama T, Matsuda T, Kimura T, Johkoh T, Tabata K, Fukuoka J, Kondoh Y. Usefulness of new diagnostic criteria for chronic hypersensitivity pneumonitis established on the basis of a Delphi survey: a Japanese cohort study. Respir Investig. 2020;58:52-8.

\section{Publisher's Note}

Springer Nature remains neutral with regard to jurisdictional claims in published maps and institutional affiliations.
Ready to submit your research? Choose BMC and benefit from:

- fast, convenient online submission

- thorough peer review by experienced researchers in your field

- rapid publication on acceptance

- support for research data, including large and complex data types

- gold Open Access which fosters wider collaboration and increased citations

- maximum visibility for your research: over $100 \mathrm{M}$ website views per year

At BMC, research is always in progress.

Learn more biomedcentral.com/submissions 